\title{
Classification of Algebraic Surfaces and Curves
}

Meirav Topol Amram

Department of Mathematics, Sce-Shamoon College Engineering, 84 Jabotinski St., Ashdod 77245, Israel

\section{Introduction}

The topology of complex algebraic surfaces is one of the central branches of modern algebraic geometry. We classify algebraic surfaces by developing invariants which differentiate between them in the moduli space of surfaces.

We embed an algebraic surface $\mathrm{X}$ in a projective space. Projecting $\mathrm{X}$ onto the projective plane, we obtain the branch curve $\mathrm{S}$ of the surface (the ramifications of the projection) which we study by degenerating $\mathrm{X}$ into a union of planes $X_{0}$. The braid monodromy technique [1,2] gives us the braid monodromy factorization of $S$, it is an invariant which distinguishes between connected components of the moduli space. Applying the van Kampen Theorem [3] we obtain the fundamental group $G$ of the complement of $S$. This group is a discrete and important invariant of the surface. For example, pairs of surfaces already exist which have the same Chern numbers and non-isomorphic Gs, see [4]

The group $G$ leads us to other groups such as the Coxeter and Artin groups. Moreover, by projecting $G$ onto the symmetric group $S n$, we obtain the fundamental group of the Galois cover of $X$. This group is the kernel of the projection and it is an important invariant of $X$. Surfaces which were already investigated are Hirzebruch surfaces, a self product of the projective line and its product with a complex torus, toric varieties and some K3 surfaces [5-12]

Having obtained the braid monodromy factorization of the curve $S$ and the fundamental group $G$, we proceed in two approaches, one based on algebra, the other one based on knot theory.

\section{Algebra}

We work with Coxeter and Artin groups. These groups turned out to be invariants of the surface as well. We consider a dual diagram $T$ of the Dynkin diagram which has a natural map onto the Coxeter group $S_{n}$ or $B_{n}$ or $D_{n}$. This idea was first introduced in [13], and further developed in [14]. We denote these Coxeter groups by $C$ (T). The group $C(T)$ has a quotient $C Y(T)$, which is a special type of the generalized Coxeter groups defined in [15]. These groups arise in the computation of certain invariants of surfaces [16]. We proved that $C Y(T)$ is isomorphic to a group $A_{H: t: n} \rtimes H$ where $n$ is the number of the vertices in $T, t$ is the number of the cycles in $T$ and $\mathrm{H}$ $€\left\{\mathrm{~S}_{\mathrm{n}} ; \mathrm{B}_{\mathrm{n}} ; \mathrm{D}_{\mathrm{n}}\right\}$. For each such $H$, there is an Artin group $A(H)$ such that $H \simeq A(H)=\left\langle<s_{1}^{2} s_{2}^{2} s_{3}^{2}, \ldots, ;, s_{n}^{2}>>\right.$ Artin groups associated with finite Coxeter groups have a topological interpretation [17]

\section{Knot Theory}

Knots and links are an alternative model of the braids in the braid monodromy factorization. We investigate the singularities appearing in the branch curve by finding these models and fixing new invariants for surfaces.

The closure of a braid is a knot, which is the topological embedding of a circle into the three-sphere, or a link, which is several embedded circles. A knot diagram is a plane embedding of a knot onto the plane together with over and under-crossing information. Two diagrams

\section{Publication History:}

Received: December 14, 2015

Accepted: February 09, 2016

Published: February 11, 2016

\section{Keywords:}

Bayesian Estimation, Maximum Entropy, Non-parametric Methods, Joint Probability Distribution

represent the same link if there is a sequence of moves taking one to the other. It is this combinatorial insight that allows one to neglect the smooth topological structure. As knots and links are often difficult to present rigorously, the description in terms of braid is often convenient for calculations. The very large undertaking of a census of invariants for relatively small knots and links has begun; however, there is still much data missing for links with more than just a few components. The fundamental problem in the knot theory is to distinguish between different knots; a possible solution lies in the construction of algebraic invariants, i.e. the knot group, the fundamental group of the complement of the knot in the three-sphere or the Alexander polynomial.

In recent years categorification of this and other knot polynomials has been constructed, i.e. knot Floer homology has been developed out of the Heegaard Floer homology of three-manifolds. Categorification has finally allowed us to answer the question of distinguishing at least the unknot (the trivial knot). Summarizing briefly the goals of the research and the applied methods, the research project combines methods from geometry, group theory, low dimensional topology, knot theory and computational methods to attack the problem of classification of surfaces. In an algebraic point of view, in [14] and [18] we developed signed diagrams, which enable us to describe quotients of some Coxeter and Artin groups as a semi direct product of a group whose invariants are the number of edges and the number of cycles of the diagram, with one of the classical Coxeter or Artin groups. We develop structures necessary to generalize these results to wider classes of Coxeter and Artin groups. In a point of view of knots, we try to characterize the braids in the braid monodromy factorization by way of local intersection points in the algebraic surfaces and categorize its building blocks; we try to give a complete list for higher multiple intersection points, and expect to obtain new local orderings in deformations of surfaces to answer various questions in algebraic geometry.

\section{Competing Interests}

The authors declare that they have no competing interests.

"Corresponding Author: Dr. Meirav Topol Amram, Department of Mathematics Sce-Shamoon College Engineering, 84 JabotinskiSt., Ashdod 77245, Israel, E-mail: meiravt@sce.ac.il

Citation: Amram MT (2016) Classification of Algebraic Surfaces and Curves. Int J Appl Exp Math 1: 104. doi: http://dx.doi.org/10.15344/ijaem/2016/104

Copyright: (c) 2016 Amram et al. This is an open-access article distributed under the terms of the Creative Commons Attribution License, which permits unrestricted use, distribution, and reproduction in any medium, provided the original author and source are credited. 


\section{References}

1. Moishezon B, Teicher M (1998) Braid group techniques in complex geometry I, Line arrangements in CP2. Contemp Math 78: 425-555.

2. Moishezon B, Teicher M (1990) Braid group techniques in complex geometry II, From arrangements of lines and conics to cuspidal curves. Algebr Geom Lect Notes Math 1479: 131-180.

3. van Kampen ER (1933) On the fundamental group of an algebraic curve. Amer J Math 55: 255-260.

4. Barth W, Peters C, Van de Ven A (1984) Compact complex surfaces. Springer, Berlin.

5. Amram M, Goldberg D, Teicher M, Vishne U (2002) The fundamental group of the Galois cover of the surface CP1 × T. Algebr Geom Topol 2: 403-432.

6. Amram M, Teicher M (2003) On the degeneration, regeneration and braid monodromy of the surface T × T. Acta Applicandae Mathematicae 75: 195270 .

7. Amram M, Teicher M (2003) The fundamental group of the complement of the branch curve of the surface $\mathrm{T} \times \mathrm{T}$. Osaka J Math 40: 1-37.

8. Amram M, Goldberg D (2004) Higher degree Galois covers of CP1 $\times$ T Algebr Geom Topol 4: 841-859.

9. Amram M, Ogata S (2006) Toric varieties \{degenerations and fundamental groups\}. Michigan Math J 54: 587-610.

10. Amram M, Ciliberto C, Miranda R, Teicher M (2009) Braid monodromy factorization for a non-prime K3 surface branch curve. Israel J Math 170: 61-94.

11. Amram M, Friedman M, Teicher M (2009) On the fundamental group of the complement of the branch curve of the second Hirzebruch surface. Topo 48: $23-40$.

12. Amram M, Friedman M, Teicher $M$ (2009) The fundamental group of the branch curve of the complement of the surface CP1 $\times$ T. Acta Mathematica Sinica 25: 1443-1458.

13. Rowen L, Teicher M, Vishne U (2005) Coxeter covers of the symmetric groups. J Group Theory 8: 139-169.

14. M. Amram, R. Shwartz, M. Teicher (2010) Coxeter covers of classical Coxeter groups. IJAC 20: 1041-1062.

15. Cameron PJ, Siedel JJ, Tsaranov SV (1994) Signed graphes, Root lattices and Coxeter groups. J Algebra 164: 173-209.

16. Teicher M (1999) The fundamental group of CP2 complement of a branch curve as an extension of solvable group by a symmetric group. Math Ann 314: 19-38.

17. Brieskorn E (1971) Die Fundamentalgruppe des Raumes der regularen Orbits einer endlichen Komplexen Spiegelungsgruppe. Invent Math 12 57-61. 